\section{Usefulness of strain elastography in the differential diagnosis of ruptured epidermal cyst and superficial abscess}

\author{
Ji Na Kim¹, Hee Jin Park', Myung Sub Kim¹, Juhee Moon', Jae Hyung Park', Eugene Kim², \\ Young Hwan $\mathrm{Kim}^{3}$
}
Departments of ${ }^{1}$ Radiology, ${ }^{2}$ Orthopaedic Surgery, and ${ }^{3}$ Nuclear Medicine, Kangbuk Samsung Hospital, Sungkyunkwan University School of Medicine, Seoul, Korea

Purpose: The aim of this study was to evaluate the usefulness of strain elastography (SE) in the differential diagnosis of ruptured epidermal cyst and superficial abscess.

Methods: This retrospective study included 34 patients with ruptured epidermal cysts and 17 patients with superficial abscesses who underwent ultrasonography (US) including SE. The SE characteristics were classified into four grades (1 to 4) according to elasticity. The largest length and height of the lesion and their ratio were evaluated on the US images. Involvement of more than half of the depth of the dermis and the presence of the submarine sign were assessed.

Results: The inter-reader agreement of US and SE findings showed excellent or almost perfect agreement. The height, length, ratio of height to length, and more-than-half-depth sign did not significantly differ between ruptured epidermal cysts and superficial abscesses for either reader (reader $1, P=0.071, P=0.129, P=0.806$, and $P=0.102$, respectively; reader $2, P=0.173, P=0.053$, $P=0.669$, and $P=0.060$, respectively). The submarine sign was significantly more frequent in ruptured epidermal cysts than in superficial abscesses (both readers, $\mathrm{P}<0.001$ ). The difference in SE scores between ruptured epidermal cysts and superficial abscesses, which are harder than ruptured epidermal cysts, was statistically significant (reader 1, $\mathrm{P}=0.046$; reader 2, $\mathrm{P}=0.028$ ).

Conclusion: The SE score and submarine sign may be useful characteristics for distinguishing ruptured epidermal cyst from superficial abscess.

Keywords: Strain elastography; Ultrasound; Epidermal cyst; Abscess

Key points: Superficial abscess has a harder nature than ruptured epidermal cyst on strain elastography. Submarine sign is a significant finding to distinguish ruptured epidermal cyst from superficial abscess.

\section{Introduction}

Epidermal cysts are benign skin lesions formed by keratinizing squamous epithelium with a granular layer, occurring as well-circumscribed nodules most often on the face, trunk, and neck [1]. Most epidermal cysts remain asymptomatic. However, if the lesions grow large enough, they can disturb the normal function of surrounding structures, become infected, or rupture into adjacent soft tissue

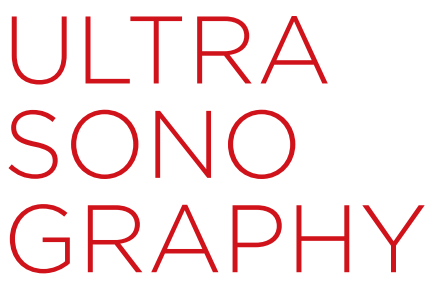

ORIGINAL ARTICLE

https://doi.org/10.14366/usg.21040 pISSN: 2288-5919 - elSSN: 2288-5943 Ultrasonography 2022;41:198-203

Received: February 23, 2021

Revised: June 3, 2021

Accepted: June 27, 2021

Correspondence to:

Hee Jin Park, MD, Department of Radiology, Kangbuk Samsung Hospital, Sungkyunkwan University School of Medicine, 29 Saemunan-ro, Jongnogu, Seoul 03181, Korea

Tel. +82-2-2001-1035

Fax. +82-2-2001-1030

E-mail: parkhiji@gmail.com

This is an Open Access article distributed under the terms of the Creative Commons Attribution NonCommercial License (http://creativecommons.org/ licenses/by-nc/4.0/) which permits unrestricted noncommercial use, distribution, and reproduction in any medium, provided the original work is properly cited.

Copyright (C) 2022 Korean Society of Ultrasound in Medicine (KSUM)

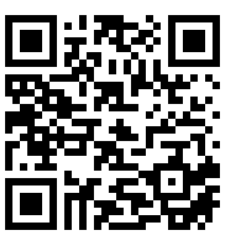

How to cite this article:

Kim JN, Park HJ, Kim MS, Moon J, Park JH, Kim E, et al.Usefulness of strain elastography in the differential diagnosis of ruptured epidermal cyst and superficial abscess. Ultrasonography. 2022 Jan;41(1):198-203. 
[2]. Ruptured epidermal cysts may result in a secondary foreign body reaction, granulomatous reaction, or abscess formation [3]. The typical imaging findings of ultrasonography (US) of a ruptured epidermal cyst are as follows: a lobulated contour; focal wall perforation; a short neck; a deep, large, protruding, mass-like portion; pericystic changes; and color Doppler signals exhibiting findings similar to those of solid masses $[3,4]$. A superficial abscess can be seen as a variably-echoic abscess cavity with a surrounding hyperechoic induration on gray-scale images [5]. In some cases, it is difficult to distinguish a ruptured epidermal cyst from a skin or subcutaneous abscess in daily practice. A superficial abscess can be defined as an abscess cavity in the skin and subcutaneous tissue resulting from a soft tissue infection. A ruptured epidermal cyst can lead to inflammation and abscess formation, and complete excision may be required for curative treatment and final diagnosis because it can recur after simple antibiotic treatment $[4,6]$. Conversely, the treatment of superficial abscesses or inflammation may involve medical treatment or drainage of pus. Differentiation of the two lesion types is important for proper treatment. Strain elastography (SE) is a widely used technique for visualizing an elastographic map in real time that is useful in evaluating the musculoskeletal system [7]. SE is helpful for differentiating epidermal cyst from other benign and malignant tumors [8]. SE analyzes tissue stiffness and provides more information about soft tissue lesions as an adjunct to conventional US imaging. In a previous study, SE showed different elasticity values between ruptured and un-ruptured epidermal cysts [6]. To the best of the authors' knowledge, no study has yet investigated the application of SE in the differential diagnosis between ruptured epidermal cyst and superficial abscess.

In the present study, the US findings, including SE, of ruptured epidermal cysts and superficial abscesses were evaluated, the usefulness of SE and other US findings in the differential diagnosis between these two lesion types was investigated.

\section{Materials and Methods}

\section{Compliance with Ethical Standards}

The ethics review board of the authors' hospital (KBSMC, IRB No. 2020-07-049) approved this study. The requirement for informed consent was waived because the study was retrospective in design.

\section{Case Selection}

A retrospective review was conducted of the US and SE data of 103 surgically confirmed ruptured epidermal cysts and superficial abscesses between May 2013 and May 2020. All patients who underwent US and surgical excision during this period were included. Cases without SE images were excluded. All ruptured epidermal cysts and superficial abscesses were diagnosed histologically. A total of 38 cases (21 men and 17 women) involving ruptured epidermal cysts and 21 patients ( 7 men and 14 women) with superficial abscesses were enrolled in this study.

\section{US Examinations}

Two musculoskeletal radiologists with 16 years and 9 years of experience, respectively, in musculoskeletal imaging performed the US examinations using a LOGIQ E9 (GE Medical System, Milwaukee, WI, USA) imaging device equipped with linear $6-15 \mathrm{MHz}$ probes and an IU22 (Philips Medical Systems, New Hartford, CT, USA) imaging device equipped with linear 5-12 MHz probes. Grayscale images and SE were obtained at the same time. The grayscale images were obtained in the longitudinal and transverse planes. SE was obtained at least twice for each lesion to collect reproducible images. The force transmitted to the lesions was adjusted appropriately according to the pressure graph or box. The rectangular region of interest (ROI) in the SE image covered the entire lesion. The relative stiffness of the lesion was displayed as a color-coded representation, ranging from red (high elasticity) to blue (low elasticity) on a continuous spectrum.

\section{Analysis of US Images}

Two musculoskeletal radiologists independently evaluated US images retrospectively, blinded to the pathology results. The largest length and height of the lesion were measured. The ratio of height to length was also calculated. The more-than-half-depth sign and submarine sign were analyzed. The more-than-half-depth sign was defined as a lesion that involved more than half the depth of the dermis (Fig. 1). The submarine sign was defined as a lesion that exhibited focal projection of the hypoechoic portion, regardless of width, height, and morphology, toward the epidermis (Fig. 2) [9].

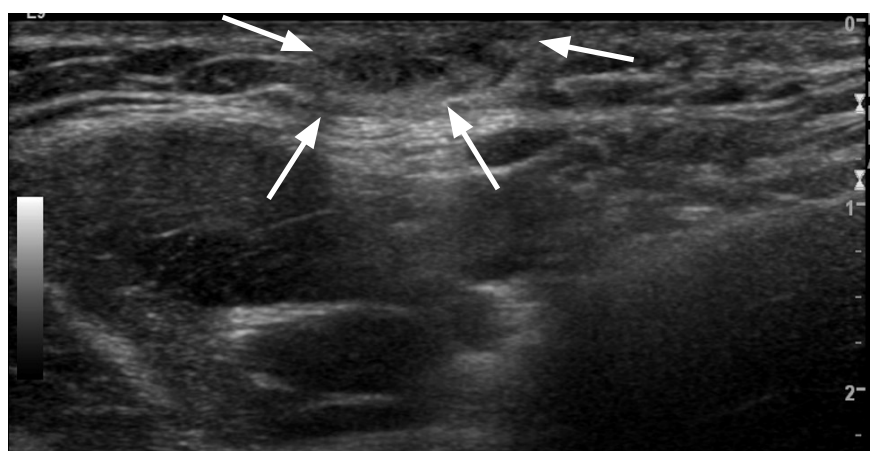

Fig. 1. More-than-half-depth sign. A 25-year-old man presented with a superficial abscess in the left axilla. The irregularly shaped hypoechoic abscess involved more than half of the depth of the dermis (arrows). 


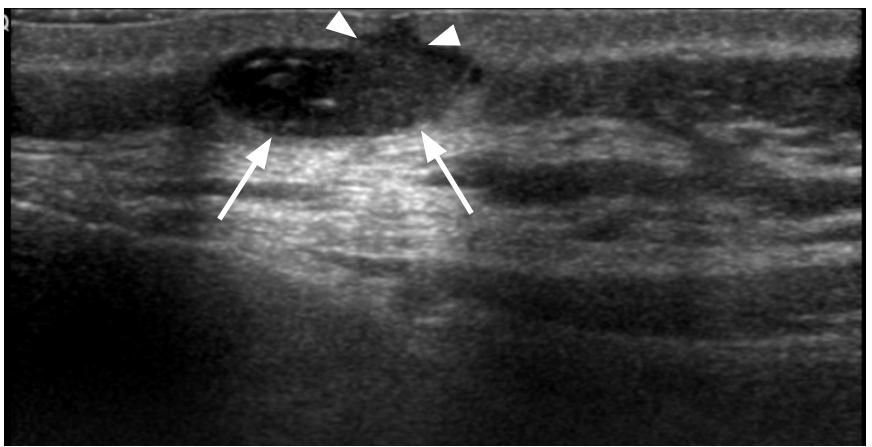

Fig. 2. Submarine sign. A 43-year-old man presented with a ruptured epidermal cyst (arrows) in the posterior neck. The focal protrusion of the hypoechoic portion (arrowhead) from the main mass into the dermis represents the submarine sign.

The SE scale was classified into four grades (1 to 4) according to elasticity (Fig. 3) [8]: score 1 (high elasticity), predominantly green to red $(>70 \%$ of the total area of the lesion), with a few small areas of blue in the ROl; score 2 (moderately high elasticity), more green than blue but not predominant (between $50 \%$ and $70 \%$ in the ROI); score 3 (moderately low elasticity), more blue than green but not predominant in the ROl; score 4 (low elasticity), predominantly blue with a few small areas of green in the ROI.

\section{Statistical Analysis}

The intra-class correlation coefficient (ICC) with a two-way randomeffects model was used for inter-reader agreement of height and length of the lesions on US images. ICC values less than 0.5 were indicative of poor reproducibility, ICC values between 0.5 and 0.75 indicated moderate reproducibility, ICC values between 0.75 and 0.9 indicated good reproducibility, and ICC values greater than 0.9 indicated excellent reproducibility [10]. Inter-reader agreement for the more-than-half-depth sign, submarine sign, and SE score between the two radiologists was analyzed using weighted kappa statistics. Kappa values of 0.00 to 0.01 were considered to indicate poor agreement; 0.01 to 0.20 , slight agreement; 0.21 to 0.40 , fair agreement; 0.41 to 0.60 , moderate agreement; 0.61 to 0.80 , substantial agreement; and 0.81 to 1 , almost perfect agreement [11]. Height, length, the ratio of height to length, and SE scores were compared between ruptured epidermal cysts and superficial abscesses using the Mann-Whitney $U$ test. The more-than-half-depth sign and submarine sign were compared using the chi-square test. Data analysis was performed using SPSS version 24.0 (IBM Corp., Armonk, NY, USA), and a P-value $\leq 0.05$ was considered to indicate statistical significance.
Table 1. Inter-reader agreement for the sonographic parameters

\begin{tabular}{lccc}
\hline \multicolumn{1}{c}{$\begin{array}{c}\text { Sonographic } \\
\text { parameter }\end{array}$} & $\begin{array}{c}\text { Inter-reader } \\
\text { agreement }\end{array}$ & $95 \% \mathrm{Cl}$ & P-value \\
\hline Height & 0.987 & $0.978-0.992$ & $<0.001$ \\
Length & 0.959 & $0.932-0.976$ & $<0.001$ \\
Height/length ratio & 0.967 & $0.944-0.980$ & $<0.001$ \\
Submarine sign & 1.000 & 1.000 & $<0.001$ \\
$\begin{array}{l}\text { More-than-half- } \\
\text { depth sign }\end{array}$ & 0.982 & $0.947-1.017$ & $<0.001$ \\
SE score & 0.973 & $0.920-1.026$ & $<0.001$ \\
\hline
\end{tabular}

ICC values for height, length, and the height/length ratio.

Kappa values for the submarine sign, more-than-half-depth sign, and SE score. $\mathrm{Cl}$, confidence interval; SE, strain elastography.

\section{Results}

The mean age of patients with ruptured epidermal cysts was 37.7 years (range, 15 to 75 years; SD, 13.9), and the mean age of patients with superficial abscesses was 38.2 years (range, 12 to 78 years; SD, 17.9). There was no significant difference in age between the two groups $(P=0.805)$. Inter-reader agreement for the sonographic parameters is shown in Table 1. Inter-reader agreement of height, length, and ratio of height to length on US exhibited excellent reproducibility. Inter-reader agreement of the more-thanhalf-depth sign, submarine sign, and SE score demonstrated almost perfect agreement. The height, length, and ratio of height to length were not significantly different between ruptured epidermal cysts and superficial abscesses for either reader (reader $1, P=0.071$, $P=0.129$, and $P=0.806$, respectively; reader $2, P=0.173, P=0.053$, and $P=0.669$, respectively). The more-than-half-depth sign was not significantly different between ruptured epidermal cysts and superficial abscesses for either reader (reader $1, P=0.102$; reader $2, P=0.060$ ). The submarine sign was significantly more frequent in ruptured epidermal cysts than in superficial abscesses (reader 1 , $\mathrm{P}<0.001$; reader $2, \mathrm{P}<0.001$ ). There were significant differences in the SE scores of ruptured epidermal cysts and superficial abscesses (reader 1, $\mathrm{P}=0.046$; reader $2, \mathrm{P}=0.028$ ) (Table 2). The percentage of score 4 masses was significantly higher in superficial abscesses than in ruptured epidermal cysts (reader $1,76 \%$ vs. $42 \%, P=0.015$; reader $2,76 \%$ vs. $40 \%, P=0.013$ ). The percentage of scores $1-3$ was significantly higher in ruptured epidermoid cysts than in superficial abscesses (reader $1,58 \%$ vs. $24 \%, P=0.015$; reader 2 , $60 \%$ vs. $24 \%, P=0.013)$. On the gray-scale images, nine of the 38 ruptured epidermal cysts were initially considered superficial abscesses, and five cases were considered to be ruptured epidermal cysts, but the second consideration was superficial abscess. Two cases were initially considered to be other soft tissue tumor-like 


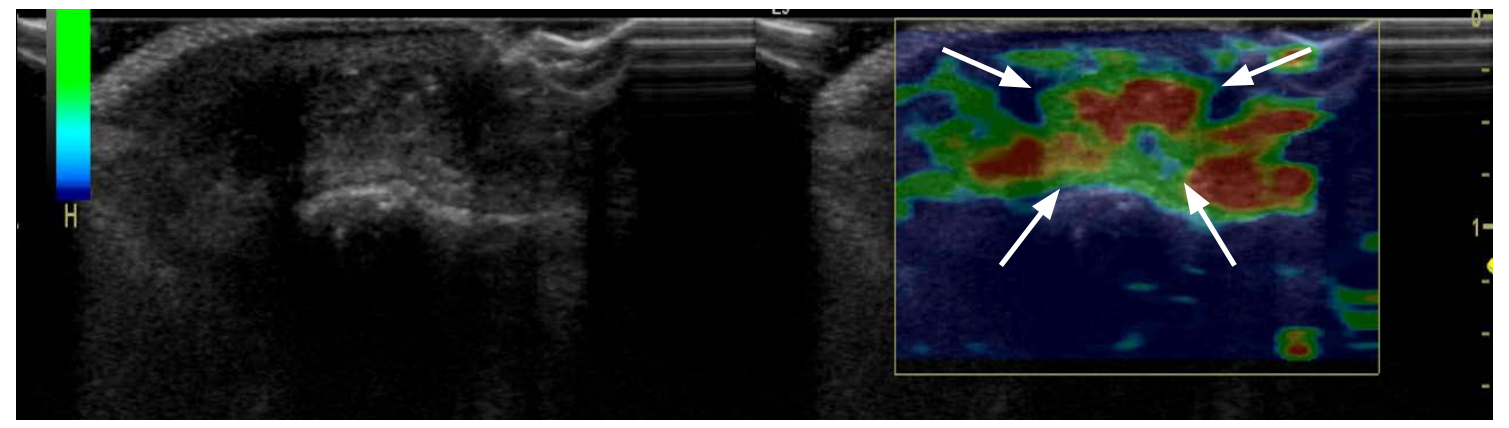

A

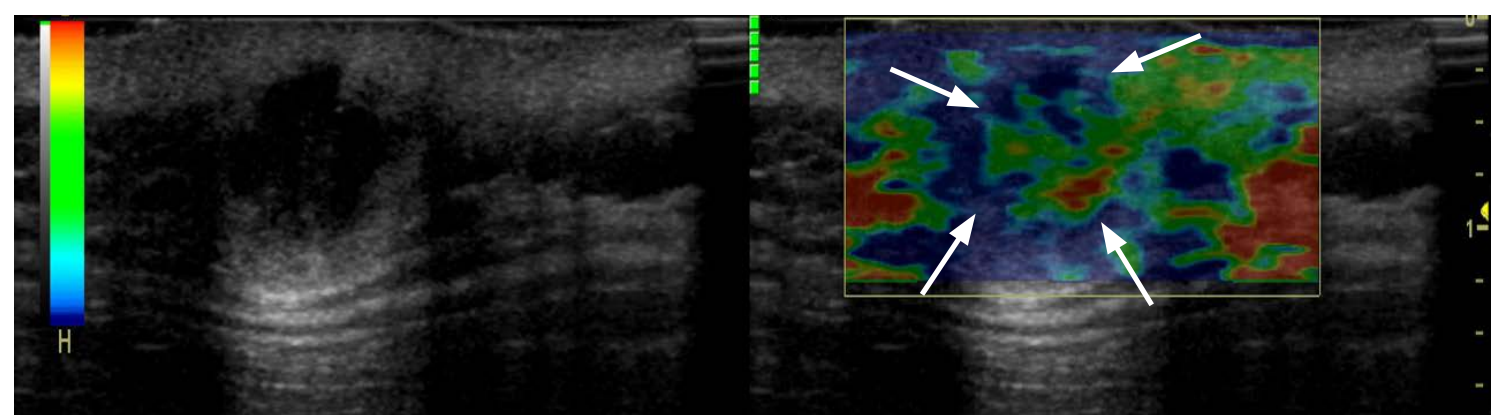

B

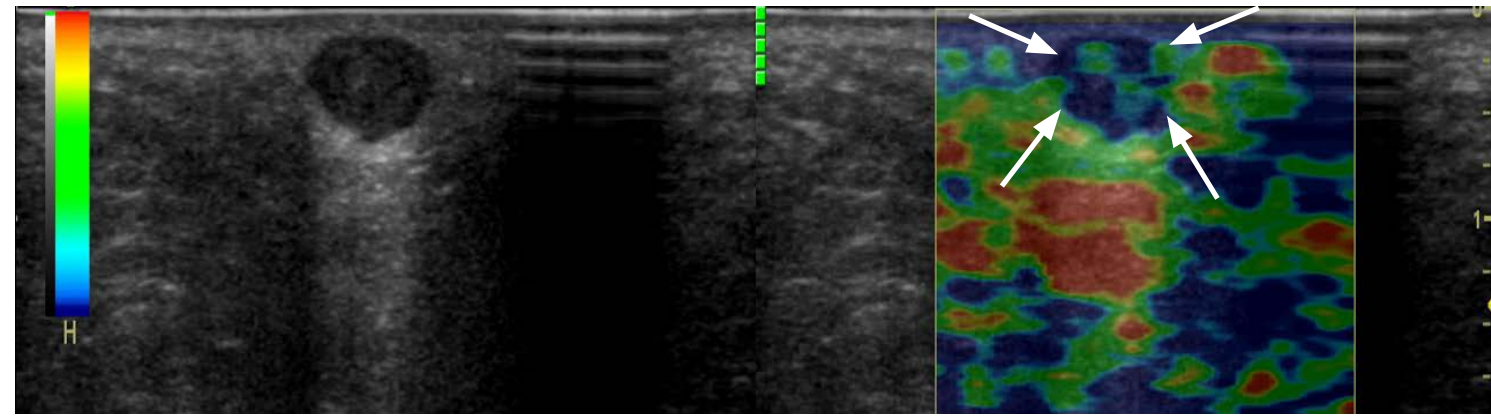

C

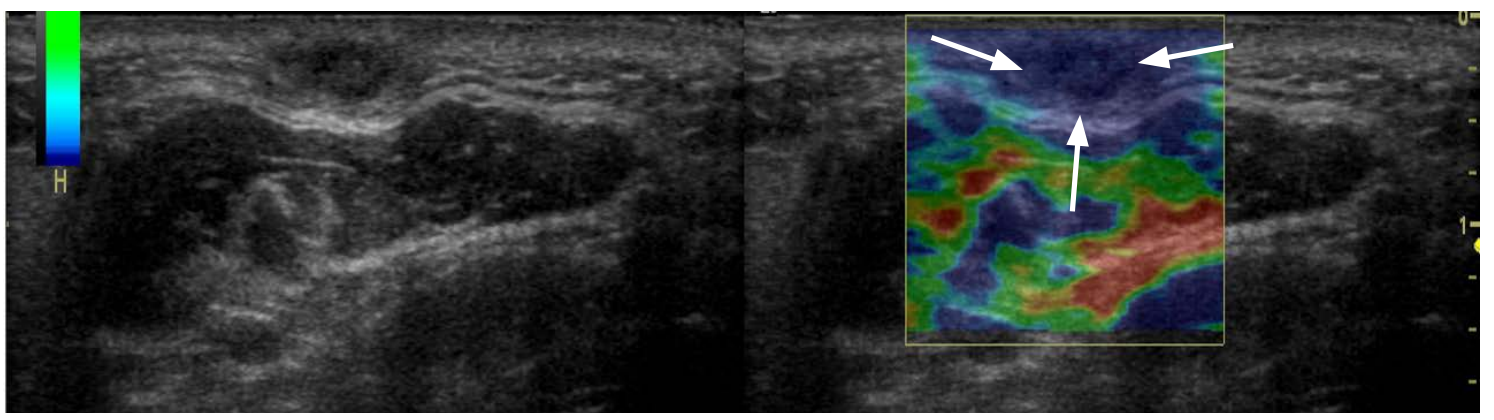

D

Fig. 3. Strain elastography scores.

A. A 56-year-old man presented with a ruptured epidermal cyst in the volar aspect of the left great toe. Strain elastography (right) exhibited green to orange colors in $>70 \%$ of the lesion (arrows). The strain elastography score was 1 . B. A 75-year-old man presented with a ruptured epidermal cyst in the posterior neck. Strain elastography (right) exhibited green to orange colors in $>50 \%$ but $<70 \%$ of the lesion (arrows). The strain elastography score was 2. C. A 55-year-old woman presented with a superficial abscess in the right buttock. Strain elastography (right) exhibited blue colors in $>50 \%$ but $<70 \%$ of the lesion (arrows). The strain elastography score was 3. D. A 40 -year-old woman presented with a superficial abscess in the left neck. Strain elastography (right) exhibited predominantly blue color in $>70 \%$ of the lesion (arrows). The strain elastography score was 4. 
Table 2. Characteristics of ruptured epidermal cysts and superficial abscesses

\begin{tabular}{|c|c|c|c|c|c|c|}
\hline \multirow[b]{2}{*}{ Diagnosis } & \multicolumn{3}{|c|}{ Reader 1} & \multicolumn{3}{|c|}{ Reader 2} \\
\hline & $\begin{array}{c}\text { Ruptured } \\
\text { epidermal cysts }\end{array}$ & $\begin{array}{c}\text { Superficial } \\
\text { abscesses }\end{array}$ & P-value & $\begin{array}{c}\text { Ruptured } \\
\text { epidermal cysts }\end{array}$ & $\begin{array}{c}\text { Superficial } \\
\text { abscesses }\end{array}$ & P-value \\
\hline Height (mm) & $6.1 \pm 3.1$ & $5.6 \pm 5.8$ & 0.071 & $6.6 \pm 3.1$ & $6.2 \pm 5.6$ & 0.173 \\
\hline Length (mm) & $14.6 \pm 7.3$ & $11.9 \pm 6.2$ & 0.129 & $16.6 \pm 8.2$ & $13.4 \pm 7.6$ & 0.053 \\
\hline Height/length ratio & $0.48 \pm 0.25$ & $0.52 \pm 0.36$ & 0.806 & $0.44 \pm 0.21$ & $0.51 \pm 0.29$ & 0.669 \\
\hline More-than-half-depth sign, n (\%) & 18/38 (47.4) & 15/21 (71.4) & 0.102 & $17 / 38(44.7)$ & 15/21 (71.4) & 0.060 \\
\hline Submarine sign, $\mathrm{n}(\%)$ & 15/38 (39.5) & $0 / 21(0)$ & $<0.001$ & $15 / 38(39.5)$ & $0 / 21(0)$ & $<0.001$ \\
\hline SE score $(1 / 2 / 3 / 4), n$ & $4 / 5 / 13 / 16$ & $2 / 2 / 1 / 16$ & 0.046 & $4 / 5 / 14 / 15$ & $2 / 2 / 2 / 15$ & 0.028 \\
\hline
\end{tabular}

$\mathrm{SE}$, strain elastography.

lesions. Eight of the nine cases incorrectly diagnosed as superficial abscess showed the more-than-half-depth sign ( $P=0.004$ and $P=0.001$ for readers 1 and 2, respectively) and no submarine sign $(P=0.101$ and $P=0.101$ for readers 1 and 2, respectively). An SE score of 4 was seen in six of the nine cases, and the SE findings were not significantly different between cases with incorrect and correct initial diagnoses $(P=0.054$ and $P=0.233$ for readers 1 and 2 , respectively). Four of the 21 cases of superficial abscesses were initially considered to be epidermal cysts on gray-scale images, and three of the 21 cases had epidermal cyst as the second differential diagnosis. They all showed the more-than-half-depth sign ( $P=0.281$ and $P=0.281$ for readers 1 and 2, respectively) and no submarine sign. On SE images, six of the seven cases showed a score of 4 and there was no significant difference between those with initially correct and incorrect diagnoses ( $\mathrm{P}=0.624$ for readers 1 and 2 ).

\section{Discussion}

The present study demonstrated that the features distinguishing ruptured epidermal cyst from superficial abscess on US and SE images were the submarine sign and SE score. Epidermal cysts may result from traumatic implantation of epidermis into the dermis or subcutaneous tissue or obstruction of hair follicles in a hair-bearing area of the body [12]. Epidermal cysts typically contain a connecting tract to the epidermal surface, also called the punctum [13]. The submarine sign may be correlated with the punctum connecting the epidermis to the epidermal cyst [9]. The submarine sign was significantly more often detected in ruptured epidermal cysts in the present study $(P<0.001)$. This sonographic feature may also be useful in distinguishing epidermal cysts from superficial abscesses, as $39.5 \%$ of ruptured epidermal cysts showed the submarine sign, and in the nine cases without the submarine sign, an incorrect diagnosis was initially made based on the gray-scale images. Another sonographic feature of epidermal cysts that differentiates them from other superficial soft tissue lesions is the more-than-halfdepth involvement of the dermis. Lee et al. [9] reported an $84 \%$ prevalence of involvement of more than half of the depth of the dermis in epidermal cysts. However, in this study, a lower prevalence (48\% and $45 \%$, each reader) of the more-than-half-depth sign was detected than in the previous study. This difference may have been caused by the exclusion of dermal involvement based only on the punctum. Although it was not statistically significantly different, the more-than-half-depth sign was detected more frequently in superficial abscesses $(71 \%)$ than in ruptured epidermal cysts. Inflammation may easily spread from the dermis to subcutaneous tissue, resulting in greater involvement of the dermis.

The differences in the height and length of the lesions between ruptured epidermal cysts and superficial abscesses were not statistically significant $(P>0.05)$. The ratio of height to length was also not significantly different between the two groups. The shape of the ruptured epidermal cysts was lobulated or protruded $[4,12]$, and the morphology of superficial abscesses can be variable. The shape or size ratio cannot be used to diagnose a ruptured epidermal cyst or superficial abscess, which is the main reason why it is difficult to distinguish between the two types of lesions on US images.

Several studies have investigated the SE findings of epidermal cysts and superficial abscesses. Patel et al. [14] reported that most epidermal cysts exhibit the feature of hardness, as shown by a characteristically blue color on SE images. Park et al. [8] reported that epidermal cysts showed lower SE scores than malignant soft tissue tumors. Park et al. [6] reported that relative hardness was significantly lower in ruptured epidermal cysts than in un-ruptured epidermal cysts on SE. This could be explained by the hypothesis that epidermal cyst contents leak toward the outside of the cyst, decreasing the pressure and hardness. Gaspari et al. [5] reported that SE accurately identified induration surrounding soft tissue abscesses. Gaspari et al. [15] also reported that asymmetry of the surrounding induration on SE was associated with therapy failure. 
In the present study, SE scores were significantly different between ruptured epidermal cysts and superficial abscesses. Superficial abscesses exhibited a higher prevalence of SE score 4. In cases with the same pathologic diagnosis, there was no significant difference in the SE score between the cases with correct and incorrect diagnoses on gray-scale images. In other words, SE is helpful in the differential diagnosis of these two diseases compared to conventional US. The natural progression of an abscess from induration to fluid collection reflects the heterogeneous nature of the abscess cavity, with tissue debris mixed in with purulence, potentially resulting in hardness on SE images [5]. Furthermore, the internal hardness of ruptured epidermal cysts decreased, revealing relatively more distinct differences in the SE score between the two groups.

There are several limitations in this study. First, the study population was small, particularly the superficial abscess group. Second, the surrounding soft tissue was not included in evaluation of SE scores in either group. Both superficial abscesses and ruptured epidermal cysts could result in surrounding induration and inflammatory changes. Therefore, both lesion types can possess a heterogeneously harder nature of the surrounding tissue. As a result, the main hypoechoic lesion was compared with the surrounding tissue. Finally, although SE is essentially an operator-dependent technique, there was almost perfect agreement of SE grading between the two readers because this study was a retrospective analysis using pre-acquired images. However, there may have been inconsistencies between the two SE systems, which could be another limitation.

In conclusion, superficial abscesses exhibited a harder nature than ruptured epidermal cysts on SE images. The submarine sign was a significant finding for distinguishing ruptured epidermal cysts from superficial abscesses. These characteristics may be helpful for an accurate diagnosis and creation of a treatment plan.

ORCID: Ji Na Kim: https://orcid.org/0000-0002-1877-9273; Hee Jin Park: https:// orcid.org/0000-0002-3135-0586; Myung Sub Kim: https://orcid.org/0000-00024346-9015; Juhee Moon: https://orcid.org/0000-0003-2854-7107; Jae Hyung Park: https://orcid.org/0000-0001-9735-4291; Eugene Kim: https://orcid.org/0000-00018569-9823; Young Hwan Kim: https://orcid.org/0000-0002-6513-3846

\section{Author Contributions}

Conceptualization: Park HJ. Data acquisition: Kim JN, Park HJ. Data analysis or interpretation: Park HJ, Kim MS, Moon J, Park JH, Kim E, Kim YH. Drafting of the manuscript: Kim JN, Park HJ, Kim MS, Park $J H$. Critical revision of the manuscript: Park HJ, Moon J, Kim E, Kim YH. Approval of the final version of the manuscript: all authors.

\section{Conflict of Interest}

No potential conflict of interest relevant to this article was reported.

\section{References}

1. Vincent $L M$, Parker LA, Mittelstaedt CA. Sonographic appearance of an epidermal inclusion cyst. J Ultrasound Med 1985;4:609-611.

2. Misner SC, Mariash SA, Alvarez G. Ruptured plantar epidermal inclusion cyst with foreign body giant cell reaction. J Foot Surg 1991;30:38-42.

3. Lee HS, Joo KB, Song HT, Kim YS, Park DW, Park CK, et al. Relationship between sonographic and pathologic findings in epidermal inclusion cysts. J Clin Ultrasound 2001;29:374-383.

4. Jin W, Ryu KN, Kim GY, Kim HC, Lee JH, Park JS. Sonographic findings of ruptured epidermal inclusion cysts in superficial soft tissue: emphasis on shapes, pericystic changes, and pericystic vascularity. J Ultrasound Med 2008;27:171-176.

5. Gaspari R, Blehar D, Mendoza M, Montoya A, Moon C, Polan D. Use of ultrasound elastography for skin and subcutaneous abscesses. J Ultrasound Med 2009;28:855-860.

6. Park J, Chae IS, Kwon DR. Utility of sonoelastography in differentiating ruptured from unruptured epidermal cysts and implications for patient care. J Ultrasound Med 2015;34:1175-1181.

7. Kim SJ, Park HJ, Lee SY. Usefulness of strain elastography of the musculoskeletal system. Ultrasonography 2016;35:104-109.

8. Park HJ, Lee SY, Lee SM, Kim WT, Lee S, Ahn KS. Strain elastography features of epidermoid tumours in superficial soft tissue: differences from other benign soft-tissue tumours and malignant tumours. $\mathrm{Br} J$ Radiol 2015;88:20140797.

9. Lee $\mathrm{DH}$, Yoon CS, Lim BJ, Lee HS, Kim S, Choi AL, et al. Ultrasound feature-based diagnostic model focusing on the "submarine sign" for epidermal cysts among superficial soft tissue lesions. Korean J Radiol 2019;20:1409-1421.

10. Cohen J. Statistical power analysis for the behavioral sciences. 2nd ed. Hillsdale, NJ: Lawrence Erlbaum Associates, 1988.

11. Koo TK, Li MY. A guideline of selecting and reporting intraclass correlation coefficients for reliability research. J Chiropr Med 2016;15:155-163.

12. Yuan WH, Hsu HC, Lai YC, Chou YH, Li AF. Differences in sonographic features of ruptured and unruptured epidermal cysts. J Ultrasound Med 2012;31:265-272.

13. Wortsman X. Common applications of dermatologic sonography. J Ultrasound Med 2012;31:97-111.

14. Patel K, Sellars ME, Clarke JL, Sidhu PS. Features of testicular epidermoid cysts on contrast-enhanced sonography and real-time tissue elastography. J Ultrasound Med 2012;31:115-122.

15. Gaspari R, Blehar D, Briones J, Dayno M. Sonoelastographic characteristics of abscess induration associated with therapy failure. J Ultrasound Med 2012;31:1405-1411. 